\title{
A Potentially Unifying Constant of Nature (Brief Note)
}

\author{
Eugene Terry Tatum ${ }^{1 *}$, U. V. S. Seshavatharam ${ }^{2}$, S. Lakshminarayana ${ }^{3}$ \\ ${ }^{1}$ Independent Researcher, Bowling Green, KY, USA \\ ${ }^{2}$ Honorary Faculty, I-SERVE, Survey No-42, Hitech City, Hyderabad, Telangana, India \\ ${ }^{3}$ Department of Nuclear Physics, Andhra University, Visakhapatnam, AP, India \\ Email: *ett@twc.com
}

How to cite this paper: Tatum, E.T., Seshavatharam, U.V.S. and Lakshminarayana, S. (2021) A Potentially Unifying Constant of Nature (Brief Note). Journal of Modern Physics, 12, 739-743.

https://doi.org/10.4236/jmp.2021.126047

Received: March 21, 2021

Accepted: May 5, 2021

Published: May 8, 2021

Copyright (c) 2021 by author(s) and Scientific Research Publishing Inc. This work is licensed under the Creative Commons Attribution International License (CC BY 4.0).

http://creativecommons.org/licenses/by/4.0/

\section{(c) (i) Open Access}

\begin{abstract}
This brief note describes a method by which numerous empirically-determined quantum constants of nature can be substituted into Einstein's field equation (EFE) for general relativity. This method involves treating the ratio $G / \hbar$ as an empirical constant of nature in its own right. This ratio is represented by a new symbol, $N_{T}$. It turns out that the value of $N_{T}$ (which is $\left.6.32891937 \times 10^{23} \mathrm{~m} \cdot \mathrm{kg}^{-2} \cdot \mathrm{s}^{-1}\right)$ is within $5 \%$ of Avogadro's number $N_{A}$, although the units are clearly different. Nevertheless, substitutions of $N_{T}$ or $N_{A}$ into the EFE, as shown, should yield an absolute value similar in magnitude to that calculated by the conventional EFE. The method described allows for quantum term EFE substitutions into Einstein's gravitational constant $\kappa$. These terms include $\hbar, \alpha, m_{e}, m_{p}, R, k_{B}, F, e, M_{U}$, and $m_{U}$. More importantly, perhaps, one or more of the many new expressions given for $\kappa$ may provide a more accurate result than $\kappa$ incorporating $G$. If so, this may have important implications for additional forward progress towards unification. Whether any of these new expressions for Einstein's field equation can move us closer to quantizing gravity remains to be determined.
\end{abstract}

\section{Keywords}

Unification, General Relativity, Quantum Theory, Einstein's Gravitational Constant, Tatum's Number, Avogadro's Number

\section{Introduction and Background}

There are a myriad of difficulties in attempting to unite gravity with the other fundamental forces of nature. Not the least of these is that our best gravity theory, general relativity, has classical deterministic features, whereas quantum theory is anti-deterministic, probabilistic, and built upon the foundation of Hei- 
senberg's uncertainty principle. To unite gravity with the other forces using these two theories, without some modifications to one or both theories, is a bit like mixing oil and water. It simply won't work. An "emulsifier" approach, successfully combining certain features of both theories, is needed.

One possible approach towards unification is to work towards quantizing gravity (i.e., "quantum gravity"), as we see with the work of string theorists. The other possible approach is to work towards gravitizing quantum theory [1], although there seems to be less progress from this direction.

This brief note points to a potentially useful way of bringing elements of both theories together by harmonizing two of their most fundamental constants ( $G$ and $\hbar)$ in the form of a ratio $(G / \hbar)$. One can then insert this ratio into Einstein's most fundamental gravity and quantum equations as shown herein.

\section{Results}

The analytic process in this brief note primarily involves substituting various constants of nature into previously-established relativity and quantum equations, in order to better see their relationships. Only simple algebra is required. No figures or tables are necessary to elucidate these relationships.

The latest available values of $G$ and $\hbar$ are [2]:

$$
\begin{gathered}
G=6.67430 \times 10^{-11} \mathrm{~m}^{3} \cdot \mathrm{kg}^{-1} \cdot \mathrm{s}^{-2} \\
\hbar=1.054571817 \times 10^{-34} \mathrm{~m}^{2} \cdot \mathrm{kg} \cdot \mathrm{s}^{-1}
\end{gathered}
$$

Their ratio, which we represent as $N_{T}$, is:

$$
G / \hbar=N_{T}=6.32891937 \times 10^{23} \mathrm{~m} \cdot \mathrm{kg}^{-2} \cdot \mathrm{s}^{-1}
$$

Interestingly, this ratio approximates (within 5\%) Avogadro's number $N_{A}$ :

$$
N_{\text {A }}=6.02214076 \times 10^{23} \mathrm{~mol}^{-1}
$$

We will take advantage of this similarity ("near-equivalence") below.

Einstein's field equation (EFE) of general relativity can be expressed as follows:

$$
G_{\mu v}+g_{\mu v} \Lambda=\left[\frac{8 \pi G}{c^{4}}\right] T_{\mu \nu}
$$

wherein $G_{\mu v}$ is the Einstein tensor, $g_{\mu v}$ is the metric tensor, $\Lambda$ is the cosmological constant, $G$ is Newton's gravitational constant, $c$ is speed of light, and $T_{\mu v}$ is the stress-energy tensor.

Notice that the bracketed coefficient of the stress-energy tensor, sometimes referred to as $\kappa$ or Einstein's gravitational constant, contains $G$. Thus, a rearrangement of relation (3) can be given as follows:

$$
G=6.32891937 \times 10^{23} \hbar
$$

And substituted in relation (5) as follows:

$$
G_{\mu \nu}+g_{\mu \nu} \Lambda=\left[\frac{8 \pi N_{T} \hbar}{c^{4}}\right] T_{\mu \nu}
$$


wherein $N_{T}$ stands for $6.32891937 \times 10^{23} \mathrm{~m} \cdot \mathrm{kg}^{-2} \cdot \mathrm{s}^{-1}$ [see relation (3)].

Thus, Newton's gravitational constant $G$ has been removed from the $\kappa$ term of the EFE and substituted by the $N_{T} \hbar$ factor. This allows Planck's reduced constant to become a part of the EFE, without sacrificing any accuracy of the mathematical expression. Furthermore, it has been shown by Seshavatharam \& Lakshminarayana [3] that the magnitude of $G$ can be expressed as:

$$
G=\frac{16 \pi^{4} m_{e}^{14} \hbar c}{\alpha^{2} m_{p}^{16}}
$$

wherein $m_{e}$ is mass of the electron, $\alpha$ is the fine structure constant, and $m_{p}$ is mass of the proton. Therefore, one can rearrange this relation as follows:

$$
\frac{G}{\hbar}=N_{T}=\frac{16 \pi^{4} m_{e}^{14} c}{\alpha^{2} m_{p}^{16}}
$$

And substitute for $N_{T}$ in relation (7) as follows:

$$
G_{\mu v}+g_{\mu v} \Lambda=\left[\frac{128 \pi^{5} m_{e}^{14} \hbar}{\alpha^{2} c^{3} m_{p}^{16}}\right] T_{\mu \nu}
$$

This should also be an accurate expression of the EFE, but now with additional quantum terms integrated to express the magnitude of $\kappa$.

Furthermore, the near-equivalence of the magnitude of $N_{T}$ and $N_{A}$ allows for the magnitude of Einstein's gravitational constant to be expressed (approximately) as:

$$
\kappa=\left[\frac{8 \pi N_{A} \hbar}{c^{4}}\right]
$$

Additionally, we know of several equivalent expressions for $N_{A}$ as follows:

$$
N_{A}=\frac{R}{k_{B}}
$$

wherein $R$ is the molar gas constant and $k_{B}$ is Boltzmann's constant.

$$
N_{A}=\frac{F}{e}
$$

wherein $F$ is the Faraday constant and $e$ is the elementary charge.

$$
N_{A}=\frac{M_{U}}{m_{U}}
$$

wherein $M_{U}$ is the molar mass constant and $m_{U}$ is the atomic mass constant.

Relations (12) thru (14) are well-known [4]. These relations are mentioned here in order to provide for additional relations (15) thru (17). Thus, Einstein's gravitational constant term $\kappa$ can also be expressed as follows:

$$
\kappa=\left[\frac{8 \pi R \hbar}{k_{B} c^{4}}\right]
$$

wherein the EFE can now incorporate the molar gas constant, Boltzmann's constant, and Planck's reduced constant. 


$$
\kappa=\left[\frac{8 \pi F \hbar}{e c^{4}}\right]
$$

wherein the EFE can now incorporate the Faraday constant, elementary charge $e$, and Planck's reduced constant.

$$
\kappa=\left[\frac{8 \pi M_{U} \hbar}{m_{U} c^{4}}\right]
$$

wherein the EFE can now incorporate the molar mass constant, the atomic mass constant, and Planck's reduced constant.

Furthermore, if one chooses to insert Newton's gravitational constant $G$ into Einstein's quantum equation for photon energy, $E=h v$, one can re-express this relation with Planck's reduced constant, by $E=2 \pi \hbar v$, and then substitute $G / N_{T}$ for $\hbar$ as follows:

$$
E=\frac{2 \pi G v}{N_{T}}
$$

Thus, Newton's gravitational constant can be worked into Einstein's most famous quantum equation. Of course, it is trivial to continue further substitutions for $N_{T}$ along similar lines as given above. Unfortunately, nothing would be gained by this approach, as this would introduce into Einstein's (and Planck's) original precise quantum formula the roughly $5 \%$ absolute magnitude error of using $N_{A}$ as a substitute for $N_{T}$. This would be an unacceptably large error in many quantum applications.

\section{Discussion}

The approach taken above with respect to substituting various empirically-determined quantum terms into the EFE may have some value, given the relative imprecision in measuring $G$ to more than 3 or 4 decimal places. It is conceivable that one or more of the $\kappa$ substitutions introduced herein [i.e., in relations (10), (11), (15), (16) and (17)], when integrated into the EFE $\kappa$ term of relation (5), could potentially improve upon the accuracy of the EFE employing $G$ alone. Of course, this remains to be determined.

\section{Summary and Conclusion}

This brief note has described a method by which numerous empirically-determined quantum constants of nature can be substituted into Einstein's field equation for general relativity. This method involves treating the ratio $G / \hbar$ as an empirical constant of nature in its own right. This ratio is represented by a new symbol, $N_{T}$. It turns out that the value of $N_{T}\left(6.32891937 \times 10^{23} \mathrm{~m} \cdot \mathrm{kg}^{-2} \cdot \mathrm{s}^{-1}\right)$ is within 5\% of Avogadro's number $N_{A}$, although the units are clearly different. Nevertheless, substitutions of $N_{A}$ into the EFE, as shown, should yield an absolute value of a similar magnitude to that calculated by the conventional EFE [i.e., relation (5)]. More importantly, perhaps, one or more of these new expressions given for Einstein's gravitational constant term $\kappa$ may provide a more accurate 
result than $\kappa$ incorporating $G$. If so, this may have important implications for additional forward progress towards unification. Whether any of these new expressions for Einstein's field equations can move us closer to quantizing gravity remains to be determined.

\section{Data Availability}

Due to the purely theoretical nature of this undertaking, no new data were generated or analyzed in support of this research.

\section{Acknowledgements and Dedications}

This paper is dedicated to Drs. Stephen Hawking and Roger Penrose for their groundbreaking work on black holes and cosmology. Dr. Tatum also thanks Dr. Rudolph Schild of the Harvard-Smithsonian Center for Astrophysics for his past support and encouragement. Author Seshavatharam is indebted to professors Shri M. Nagaphani Sarma, Chairman, Shri K.V. Krishna Murthy, founder Chairman, Institute of Scientific Research in Vedas (I-SERVE), Hyderabad, India and Shri K.V.R.S. Murthy, former scientist IICT (CSIR), Govt. of India, Director, Research and Development, I-SERVE, for their valuable guidance and great support in developing this subject.

\section{Conflicts of Interest}

The authors declare no conflicts of interest regarding the publication of this paper.

\section{References}

[1] Howl, R., Penrose, R. and Fuentes, I. (2019). New Journal of Physics, 21, Article ID: 043047. https://doi.org/10.1088/1367-2630/ab104a

[2] Mohr, P.J., Newell, D.B. and Taylor, B.N. (2020) Metrologia, 55, 125-146.

[3] Seshavatharam, U.V.S. and Lakshminarayana, S. (2021) International Journal of Physical Research, 9, 42-48.

[4] Wikipedia Contributors (2021) Avogadro Constant. Wikipedia, The Free Encyclopedia. Web. 29 Mar. 2021. 